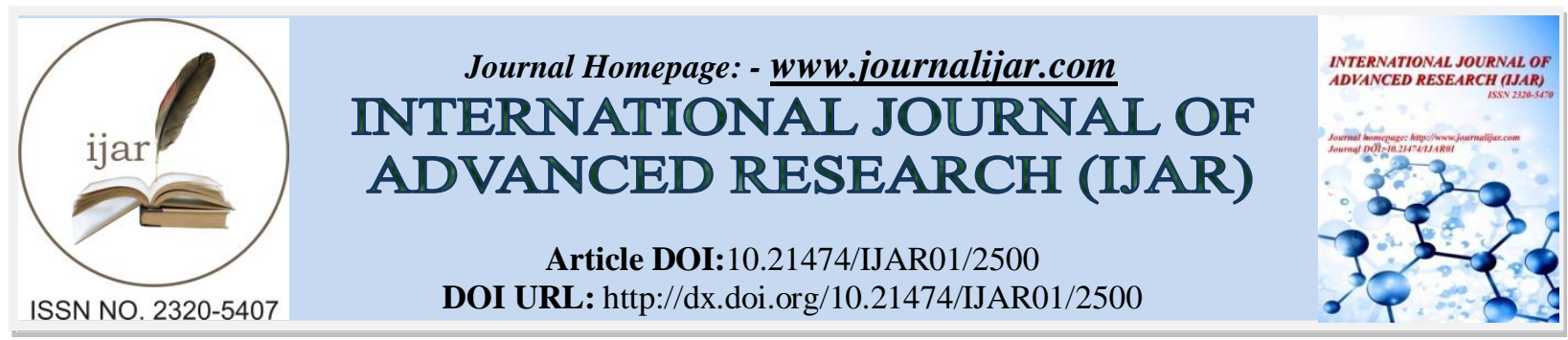

RESEARCHARTICLE

\title{
A SURVEY ON THE AWARENESS OF PATIENTS, VISITORS AND COMPANIONS ABOUT ISOLATION PRECAUTIONS.
}

\begin{abstract}
Nabeela AL-Abdullah ${ }^{1}$ BSc, MSc,PhD, Bashayr GS Alamri ${ }^{2}$, Abeer MR Daghistani ${ }^{2}$, Elaf SM Altwairqi ${ }^{2}$, MarwahHMHakami ${ }^{2}$, Abaad A AL Mutairi ${ }^{2}$, GhadiD Mashat ${ }^{2}$, Wajd A Alotaibi ${ }^{2}$, Amal AZahrani ${ }^{2}$, Raneen M Abousaadah ${ }^{2}$, HadeelM Khoj ${ }^{2}$, ElafAAlrehaili ${ }^{2}$, Rehab AAlshamrani ${ }^{2}$, Ebtisam MF Alhawsawi ${ }^{2}$, Hawazin A Fallatah ${ }^{2}$ and NedaaFA Alyami ${ }^{2}$.

1. Assistant professor and clinical epidemiology consultant. Department of Nursing, King Abdulaziz University, Jeddah, Saudi Arabia.

2. Medical intern,Kingabdulaziz University, Jeddah, Saudi Arabia.
\end{abstract}

\section{ManuscriptInfo}

Manuscript History

Received: 25 October 2016

Final Accepted: 23 November 2016

Published: December 2016

Key words:-

Isolation causes, visitors attitudes ,

Isolation percussion, prevention

\section{Abstract}

Background:-Successful infection control requires a multidisciplinary team, which includes patients, healthcare workers and patients' visitors. Transmission of multidrug-resistant organisms are always applied by health care staff and not by patient's visitors, in spite of their important role in infection transmission. The aim of this study is to assess the knowledge of patients' families/ visitors about a patient's isolation status.

Method:-This cross-sectional study was done during November 2015 in King Abdul Aziz University Hospital. By distributing a questionnaire consist of 4 parts.

Result:-Fifty two responses were collected during November 2015,of which $58 \%$ companions and $42 \%$ visitors .From 52 participants only $24(46.2 \%)$ knew about isolation, in non-isolation units $71.8 \%$ of visitors did not know if her/his patient was isolated and the other $28.2 \%$ reported knowing about isolation from either a doctor or a nurse, while all the $13(100 \%)$ visitors to isolation units know about it with significant difference $(\mathrm{p}<0.0001)$.

Conclusion:-This study focused on the importance of communication with patients' visitors. The level of education of visitors and companions plays an important role in their awareness of their right to know about patients' safety. The authors recommend that all visitors regardless of their patient's unit should be educated in simple language about the need for isolation.

\section{Introduction:-}

Successful infection control requires a multidisciplinary team, which includes patients, healthcare workers and patients' visitors. Transmissionof multidrug-resistant organisms are always applied by health care staff and not by patient's visitors ${ }^{1}$, in spite of their important role in infection transmission ${ }^{2}$. About 100,000 invasive methicillin 
resistant Staphylococcus aureus (MRSA) infections occur annually. It is the most common complication of acute hospitalization and it might be the leading cause of human death ${ }^{3,4}$.

In order to decrease and prevent the incidence of infectious diseases and transfer from person to person, there is need for teaching patients' visitors and families about isolation global cautions and showing them how to use personal protective equipment (PPE) in appropriate way as used by health providers These precautions be adhered strongly in the cases of drug-resistant Gram-negative bacteria and enteric pathogens with diarrhea . ${ }^{1}$

The Center for Disease Control and Prevention recommends the use of isolation precautions for HCW. The guidelines mentioned that visitors who are in close contact with the patients may transmit infection to other patients ${ }^{5}$. Oneof the simplest measures in preventing nosocomial infections is hand hygiene ${ }^{9}$. Moreover, promotion of hand hygiene is considered a major challenge in infection control ${ }^{6,7}$.

In Thailand study, the authors reported lower level of commitments among health providers \& patients' visitors towards hand- hygiene prior touching patients. The main three reasons for lack of commitments : primacy of patients' needs , oblivion and irritation of skin. ${ }^{8}$

On the other hand, the importance of airborne isolation awareness for example is required due to the increment of incidence of tuberculosis in Saudi Arabia particularly9.

The aim of this study is to assess the knowledge of patients' families/ visitors about a patient's isolation status.

\section{Method:-}

The research project was done in November 2015, based on a cross-sectional study. The sample of this study is a random sample. It was collected from different units at King Abdul Aziz University Hospital (KAUH) between 1 and 5 November. The involved units were: isolation, medical, surgical and pediatrics, both male and female. The questionnaire was designed in Arabic form and divided into 4 parts: patient information (e.g. patient diagnosis, transfer from another department or another hospital), visitor information (e.g. age - sex- nationality - education level), visitorinformation about isolation (e.g. awareness, types of isolation and their precautions), visitorbehavior with isolated patients (e.g. are they willing to comply or not if they know about isolation). The questionnaire was reviewed by our supervisor, validity confirmation done and data collection took place.

\section{Results:-}

During the study period, there were 52 responses, of which $58 \%$ companions and $42 \%$ visitors and the majorityof them were aged less than 60 . The majority $98 \%$ spoke Arabic language, $11 \%$ were not educated, $23 \%$ had been in elementary school and the remaindershave attended intermediate school and above. From 52 participants only 24 (46.2\%) knew about isolation , in non-isolation units $71.8 \%$ of visitors did not know if her/his patient was isolated and the other $28.2 \%$ reported knowing about isolation from either a doctor or a nurse, while all the 13 (100\%) visitors to isolation units know about it with significant difference $(\mathrm{p}<0.0001)$. (figs1\& 2 ). Among the 24 responsesthere was a variety in reporting isolation signs(Fig 3), half of them $50 \%$ reported infectious diseases as reason for isolation and 33.3\% stated bacterial organism . (Fig 4) Concerning Type of isolation and better ways of prevention , 29.2\% stated contact and airborne equally as types of isolation and 50.2\% chose mask as preventing way. (Figs 5\&6) Regarding complication and more liable groups, Pneumoniawas chosen from $25 \%$ of the participants and more than third choose children and elderlyas risky groups . (Figs 7\&8)5 .The majority (49-94.2\%) stated their right to know about isolation.. The compliance of families with personal protective equipment (PPE) when they know their patients were in isolation was $96 \%$. The majority of visitors who took the precautions were educated in intermediate and high schools. 
Figure 1:-Level of awareness among isolation and non-isolation unit

Level of awareness among isolation \& non isolation units visitors

100

30

- Non Isolation Units

Isolation Unit

Figure 2:- Level of awareness regarding the units

\section{Level of awarness regarding the units}

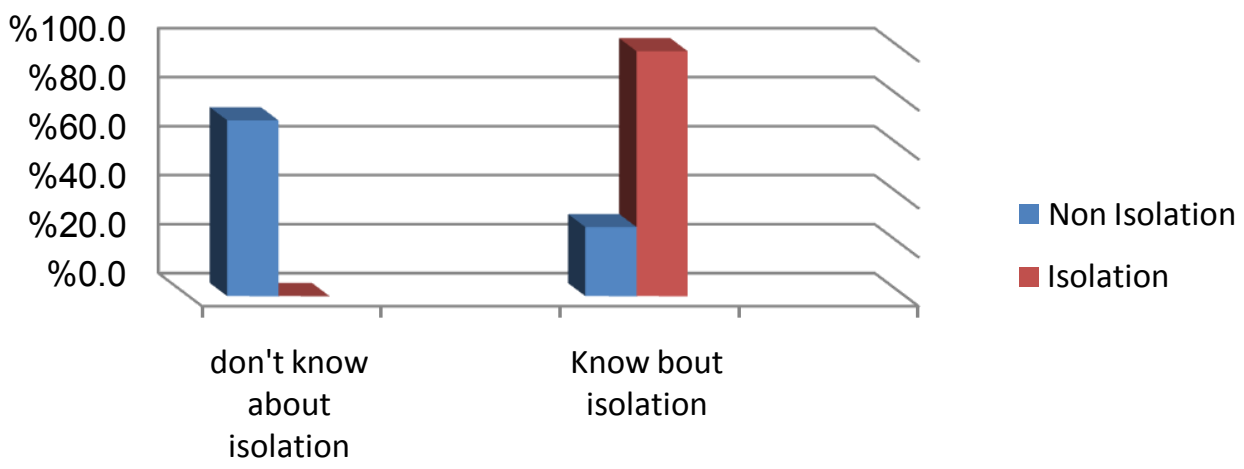

Figure 3:-Isolation signs

\section{Isolation signs}
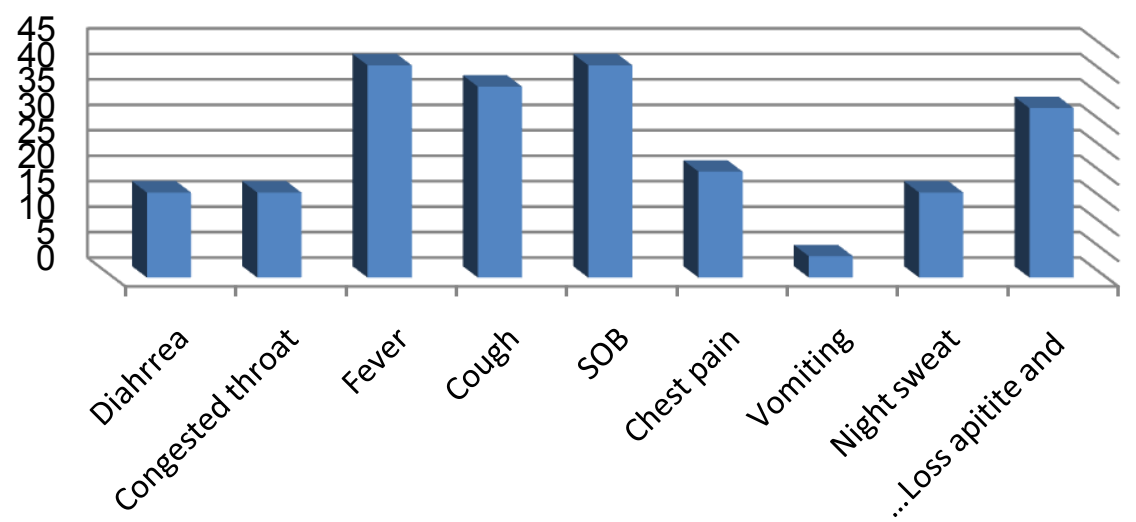
Figure 4:-Cause Of Isolation

\section{Cause Of Isolation}

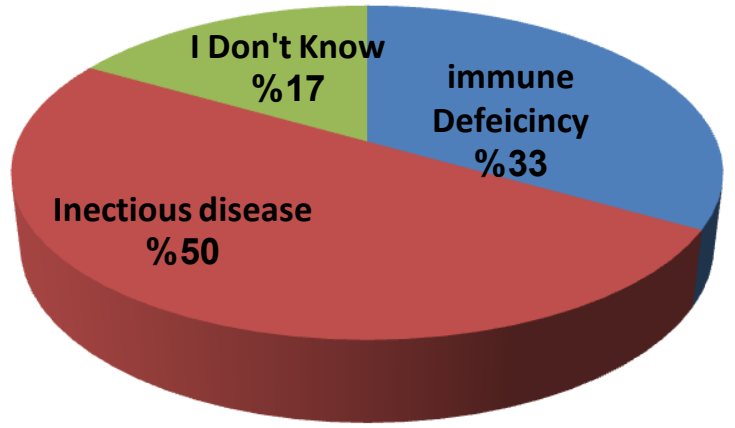

Figure 5:-Type Of Isolation.

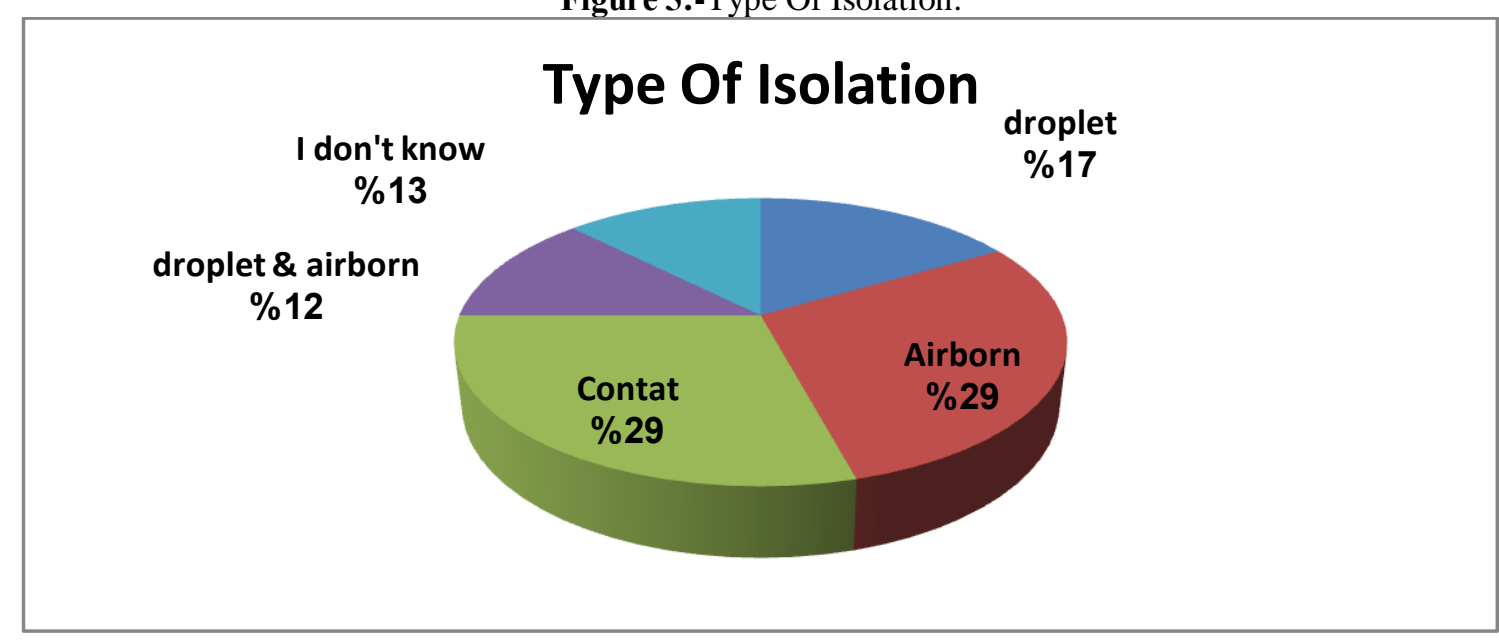

Figure 6:-Complication

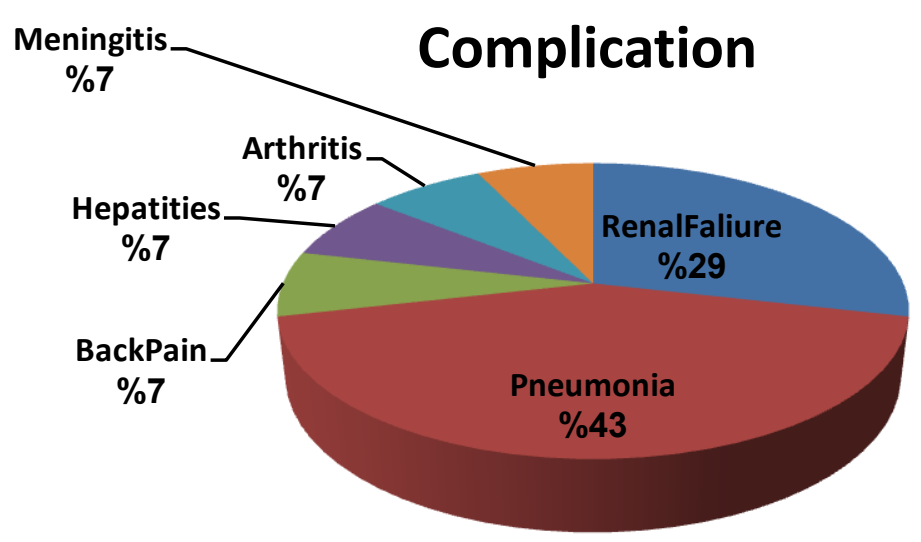


Figure 7:-Preventing ways.

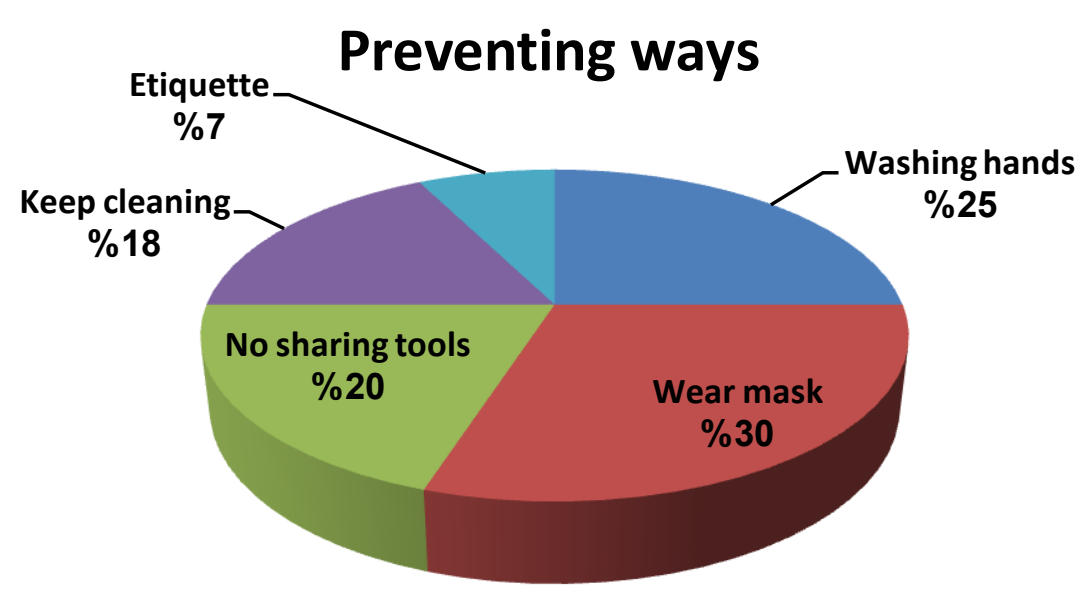

Figure 8:-More liable group

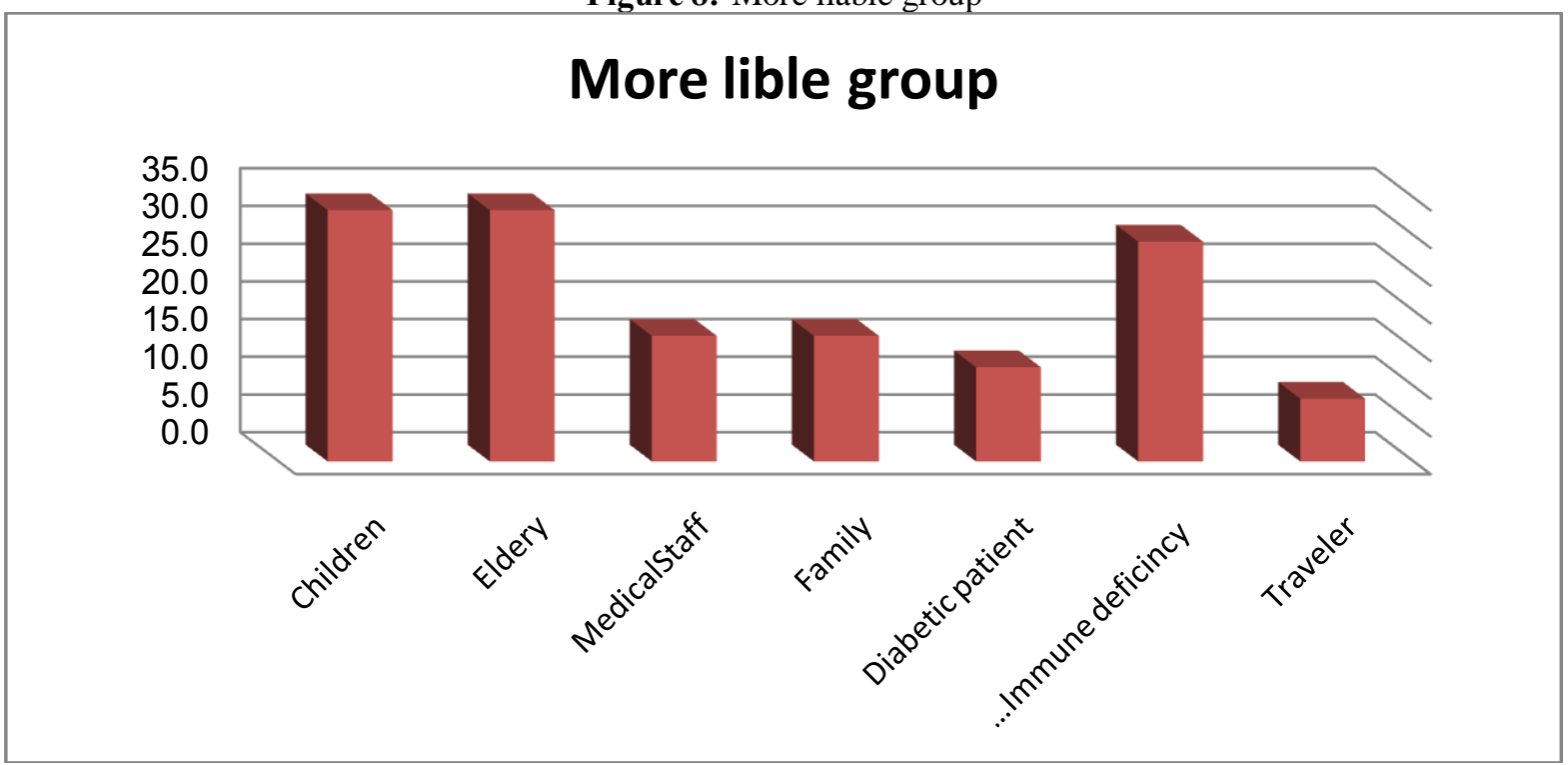

\section{Discussion:-}

Based on the current results from 52 responses; $73 \%$ of them were female, $98 \%$ less than 60 years old and $88 \%$ were educated. The survey shows substantial variation in the awareness of visitors whether patients were in isolation unit or not. More than the half of visitors did not know if her/his patient was isolated and all of them were from nonisolation units, the visitors of non-isolation were asked if they know the reason for isolation and 23\% said 'yes'. In contrast a survey done in West Virginia University (among contact isolation patients only) with 36 participants showed that $92 \%$ of visitors knew why their patients were in isolation ${ }^{1}$. In the current our study, regarding the questions of how did they know about isolation the majority $(54.5 \%)$ reported that they heard about the isolation status from staff, $(31.8 \%)$ from isolation signs, and (13.6\%) from patients. $50 \%$ of the visitors who know the reason said it was an infectious cause, (36.4\%) said it had an immunodeficiency cause, and (13.6\%) did not know the cause. The adherence of visitors to PPE when they knew that their patients were in isolation was 96\%. Several studies demonstrate that visitors' adherence to PPE varied from "very low" to $97 \%{ }^{10}$. One of the studies was conducted in two physically connected tertiary care hospitals among 288 participants and demonstrated that the most common cause of low compliance with contact isolation precautions is poor hand hygiene before entering the room ${ }^{11}$.Another study was done in USA about (Compliance With Routine Use of Gowns by Healthcare Workers and Non-HCW Visitors on Entry Into The Rooms of Patients Under Contact Precautions) showed that the compliance with routine 
precautions use in visitors of ICUs were more than visitors of general wards, this study was observed for 392 visitors ${ }^{12}$.

For decades , there was a global warning indicate the need for avoiding any direct contact with patients' secretions by wearing gloves goggles, and using Medical masks to protect nose, mouth and eyes, in 1987 these global precautions were modified and organized among a group of rules named "body substance isolation". In 1996 all these cautions were substituted by standard\& advanced cautions, and regarding these new cautions all the literature reviews now recommend the using of PPE in all health facilities. ${ }^{5}$

There are many limitations in current study. The time was limited, the sample size was small, the ICUs visitors were not included in the study and the study was conducted in one hospital; all of which make it difficult to generalize the findings.

\section{Conclusions:-}

This study focused on the importance of communication with patients' visitors. The majority of visitors and companions did not know about their patient's isolation and the reason for it. One of the causes was that the information was written in the English language only. The isolation unit visitors and companions were more educated about isolation and its precautions while non-isolation units' visitors were less educated. Thus, the authors recommend that all visitors regardless of their patient's unit should be educated about the need for isolation. The level of education of visitors and companions plays an important role in their awareness of their right to know about patients' safety. Hence, we recommend that educational material for all population levels should be presented in simple language.

\section{References:-}

1. Roidad, N. and R. Khakoo (2014). "Knowledge and attitudes of visitors to patients in contact isolation." American journal of infection control42(2): 198-199.

2. Banach, D. B., et al. (2015). "Infection control precautions for visitors to healthcare facilities." Expert review of anti-infective therapy13(9): 1047-1050.

3. Halcomb, E. J., et al. (2008). "The role of patient isolation and compliance with isolation practices in the control of nosocomial MRSA in acute care." International Journal of Evidence-Based Healthcare6(2): 206-224.

4. Klevens, R. M., et al. (2007). "Invasive methicillin-resistant Staphylococcus aureus infections in the United States." Jama298(15): 1763-1771.

5. Siegel, J. D., E. Rhinehart, et al. (2007). "2007 guideline for isolation precautions: preventing transmission of infectious agents in health care settings." American journal of infection control35(10): S65-S164.

6. Boyce, J. M. (1999). "It is time for action: improving hand hygiene in hospitals." Annals of internal medicine130(2): 153-155.

7. Goldmann, D. and E. Larson (1992). "Hand-washing and nosocomial infections." New England Journal of Medicine327(2): 120-122.

8. Patarakul, K., et al. (2005). "Cross-sectional survey of hand-hygiene compliance and attitudes of health care workers and visitors in the intensive care units at King Chulalongkorn Memorial Hospital." JOURNALMEDICAL ASSOCIATION OF THAILAND88: S287.

9. Abouzeid, M. S., et al. (2012). "Tuberculosis trends in Saudis and non-Saudis in the Kingdom of Saudi Arabiaa 10 year retrospective study (2000-2009)." PloS one7(6): e39478.

10. Kang, J., D. J. Weber, et al. (2014). "Survey of North Carolina hospital policies regarding visitor use of personal protective equipment for entering the rooms of patients under isolation precautions." Infection Control \& Hospital Epidemiology35(03): 259-264.

11. Semmelweis, I. (1988). "The etiology, concept, and prophylaxis of childbed fever." Buck C, Llopis A, Najera E, Terris M. The challenge of epidemiology. Issues and selected readings. Scientific Publication(505): 46-59.

12. Manian FA, Ponzillo JJ (2007). "Compliance With Routine Use of Gowns by Healthcare Workers and NonHCW Visitors on Entry Into The Rooms of Patients Under Contact Precautions." Infect Control Hosp Epidemiol. 2007 Mar;28(3):337-40. Epub 2007 Feb 20 